\title{
Treatment of Pulmonary Vein Stenosis after Percutaneous Ablation of Atrial Fibrillation
}

\author{
Alvaro Valentim Lima Sarabanda, Leonardo Cogo Beck, Luis Gustavo Gomes Ferreira, Wagner Luis Gali, Fernando Melo \\ Netto, Guilherme Urpia Monte \\ Instituto de Cardiologia do Distrito Federal - Fundação Universitária de Cardiologia (FUC), Brasília, DF - Brazil
}

This case report describes a patient with persistent atrial fibrillation (AF) submitted to radiofrequency catheter ablation of AF through the pulmonary vein antrum isolation technique, who developed a severe stenosis of the left superior pulmonary vein (LSPV), without presenting any symptoms. The diagnosis of the PV stenosis was made by a routine postprocedure computed tomography scan of the left atrium, and the patient was subsequently submitted to angioplasty with stenting of the LSPV, resulting in the normalization of pulmonary perfusion, as seen by ventilation/perfusion scan made three months after the LSPV angioplasty.

\section{Case report}

A 50-year-old male patient, with hypertension and persistent atrial fibrillation (AF) refractory to the antiarrhythmic treatment, was submitted to radiofrequency catheter ablation of AF through the extra-ostial (antrum) isolation of the pulmonary veins (PV), guided by intracardiac echocardiography1. During this procedure, all four PV antra were isolated individually by delivering radiofrequency energy (catheter with an 8-mm tip, 30-50 W, $50^{\circ} \mathrm{C}$ ) around a circular decapolar catheter positioned at the PV antrum. Radiofrequency application was immediately interrupted when microbubbles were detected by the intracardiac echocardiography1. At the ambulatory follow-up, the patient remained asymptomatic and received sotalol and warfarin. A routine multislice computed tomography $(\mathrm{CT})$ scan of the left atrium was made at six months after the AF ablation, and showed severe stenosis of the ostium of the left superior pulmonary vein (LSPV) (Figures $1 \mathrm{~A}$ and $1 \mathrm{C}$ ), with an area of heterogeneous opacity in the periphery of the left superior pulmonary lobe, compatible with pulmonary infarction. A ventilation/perfusion scan was performed to evaluate the functional significance of the PV stenosis, and showed a severe perfusion deficit in the left superior pulmonary lobe.

\section{Key Words}

atrial fibrillation; catheter ablation; constriction, pathologic; pulmonary veins.

Mailing address: Alvaro Valentim Lima Sarabanda•

AOS 02 Bloco B apto 604 - 70660-022 - Brasília, DF - Brazil

E-mail: saraband@uol.com.br

Manuscript received April 23, 2009; revised manuscript received June 26, 2009; accepted August 07, 2009.
The patient was submitted to a percutaneous intervention of the LSPV stenosis, guided by the transesophageal echocardiography. At the start of the procedure, a pulmonary artery wedge angiography was performed, with an angiographic catheter positioned in the superior branch of the left pulmonary artery to visualize the anatomy of the affected PV and locate its ostium. After a transseptal puncture, the catheterization of the LSPV was carried out and a retrograde pulmonary venous angiography was performed. Initially, a balloon angioplasty of the LSPV was performed (Ultra soft SV 6.0 × $20 \mathrm{~mm}$, Boston Scientific), with a significant improvement in vessel lumen and flow, although an important residual lesion remained. Subsequently, stenting (Express LD $7.0 \times 17$ mm, Boston Scientific) of the LSPV was performed. The stent diameter was chosen based on the reference diameter of the vessel proximal to the stenosis. The stent length was chosen to fully span the stenosis and to avoid excessive protrusion into the left atrium and crossing of branch points distal to the stenosis. Following angioplasty with stenting of the LSPV ostium, the angiography of the affected PV showed the re-establishment of the pulmonary venous flow, with no residual obstructions and no occlusion in the distal branches (Figure 2).

The ventilation/perfusion scan showed normalization of the pulmonary perfusion at three months. The CT scan of the left atrium, repeated 4 months after the angioplasty, showed that the stent was well-positioned at the LSPV ostium, with no evidence of obstruction (Figures 1B and 1D), and showed a significant decrease in the opacity area at the left superior pulmonary lobe. At the 8-month follow-up after the angioplasty, the patient remained asymptomatic, using warfarin and clopidogrel.

\section{Discussion}

Catheter ablation for AF is an effective treatment for patients with symptomatic AF refractory to therapy with antiarrhythmic drugs and has been increasingly performed worldwide2. Approximately $76 \%$ of the patients with AF who underwent catheter-based radiofrequency ablation remain symptom-free for a period of one year, in comparison to only $19 \%$ of patients treated with standard antiarrhythmic drugs in the same period ${ }^{3}$. However, this is not a risk-free procedure, with stenosis or occlusion of one or more pulmonary veins being one of its potential complications ${ }^{4-6}$.

The true incidence of PV stenosis following AF ablation is still a controversial issue in medical literature, with incidence 


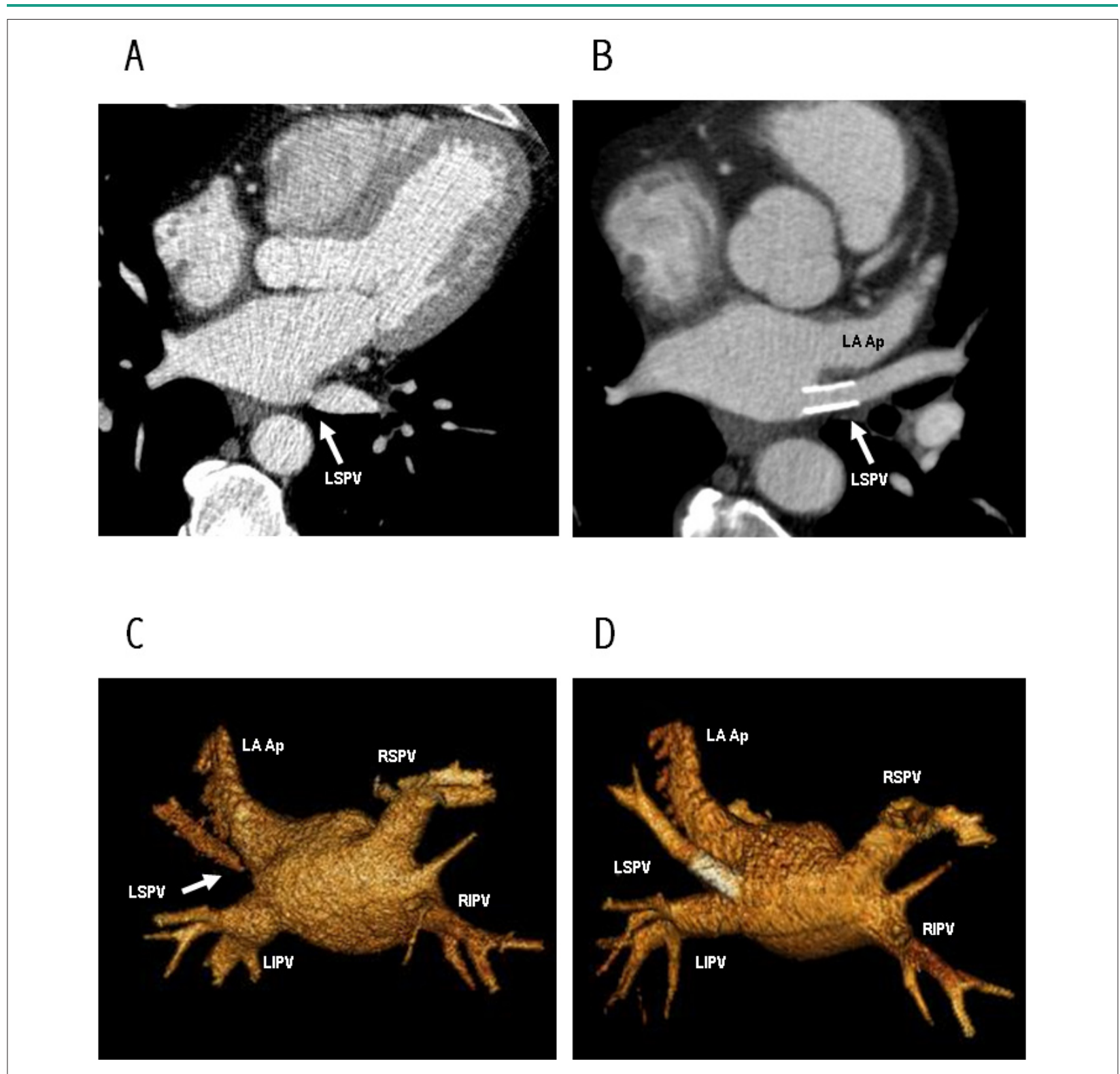

Figure 1 - A -CT scan showing severe stenosis of the LSPV ostium following AF ablation; B - CT scan following angioplasty with stenting of the LSPV ostium; C - Threedimensional CT reconstruction of the left atrium and of the PVs showing severe stenosis of the LSPV ostium following AF ablation; D - Three-dimensional CT reconstruction following angioplasty with stenting of the LSPV ostium. CT: computed tomography; LA ap: left atrial appendage; LIPV: left inferior pulmonary vein; LSPV: left superior pulmonary vein; RIPV: right inferior pulmonary vein; RSPV: right superior pulmonary vein.

rates ranging from $1.3 \%$ to $15.6 \%$ in patients undergoing PV isolation ${ }^{2,4}$. There is a perception among professionals who perform this procedure that the incidence of PV stenosis has decreased as the procedure has developed over the past few years, especially after the introduction of new ablation strategies such as PV antrum isolation ${ }^{4}$ and the use of three-dimensional mapping systems ${ }^{6}$. However, even with the use of these new ablation strategies, this complication continues to be reported ${ }^{4,6}$.

In the present case report, several factors might have contributed to the occurrence of the LSPV stenosis, and among them, the individual isolation of the ipsilateral antrum of the PV, which is associated with an increased risk of PV stenosis ${ }^{6}$. Another relevant factor could be the unintentional delivery of radiofrequency energy within the ostium of the LSPV, as a result of the narrowness of the isthmus between the orifices of the LSPV and the left atrial appendage, as seen in our patient by means of the CT scan ${ }^{6}$ (Figures 1B, 1C and 1D). Finally, it must be emphasized that during the procedure, we were very careful to limit the power of the radiofrequency energy delivered (30-50 W, $50^{\circ} \mathrm{C}$ ), always interrupting the application of radiofrequency when we detected the formation of microbubbles by the intracardiac echocardiography. However, it is important to 


\section{Case Report}

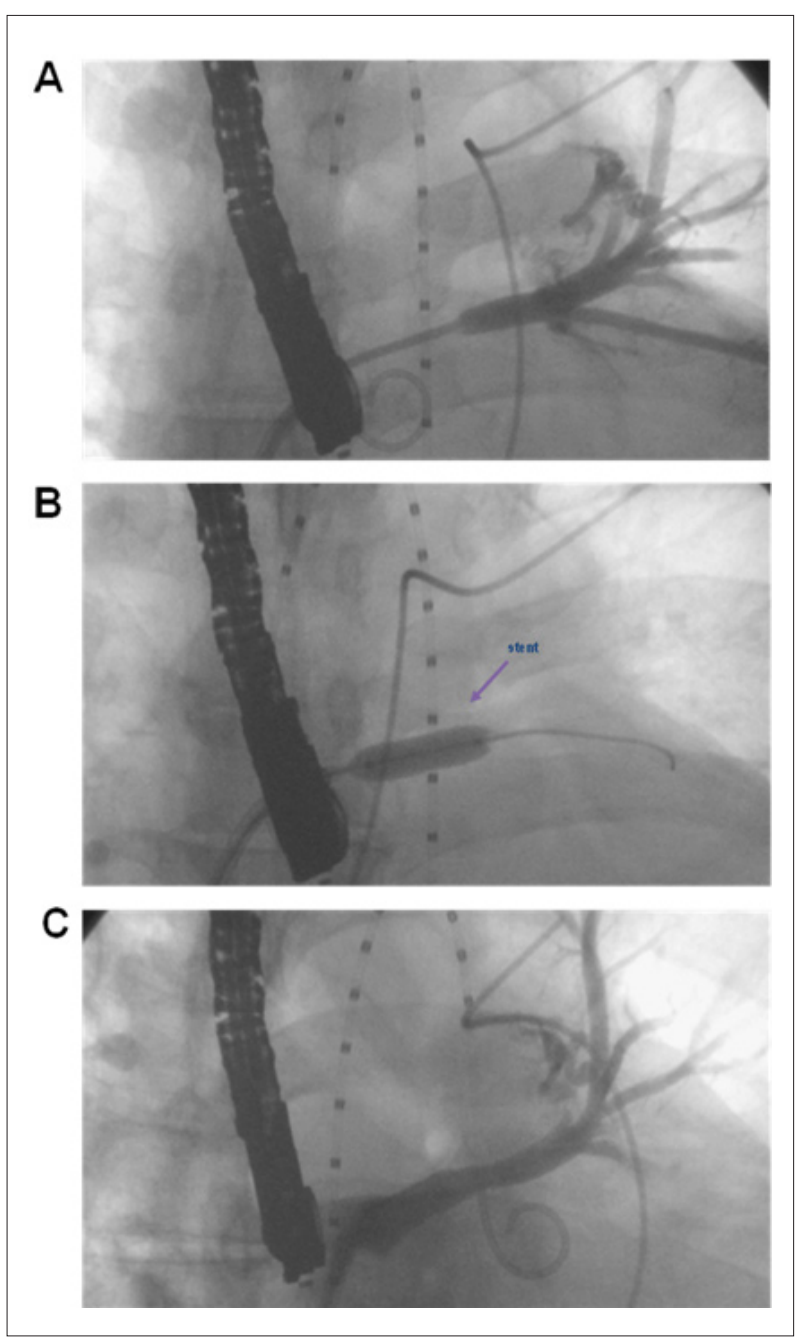

Figure 2 - $A$ - Retrograde angiography of the LSPV showing severe stenosis at its ostium; B - Stenting (Express LD $7.0 \times 17 \mathrm{~mm}$ ) of the LSPV ostium; $C$ - Angiography following LSPV stenting showing re-establishment of PV flow, with no residual obstructions and no occlusion of distal branches. The transesophageal echocardiography transducer can be seen on the left of each image.

mention that even with the use of such strategy, an excessive thermal damage can still occur at the ostium of the PV, as reported by experimental studies ${ }^{7}$.

From a clinical point of view, almost all patients with mild ( $<50 \%$ ) or moderate stenosis $(50-70 \%$ ) of the PV do not present symptoms $\mathrm{s}^{4,5}$. The occurrence of respiratory symptoms (coughing, hemoptysis and dyspnea) is associated with severe stenosis of a single PV (> 70\%) or to the involvement of multiple PV ${ }^{4,5}$. However, it is also known that patients with severe stenosis or occlusion of a single PV can remain asymptomatic ${ }^{4,5,8}$, as observed in the present case report. In this context, although still controversial, the strategy of systematically screening for PV stenosis with CT scan or magnetic resonance image for all patients undergoing PV isolation, regardless of symptom manifestation, is fully justified by the present case. In asymptomatic patients, the failure or delay in recognizing PV stenosis, with the consequent interruption of the anticoagulation after the procedure can result in thrombotic occlusion of the PV, with adverse effects on pulmonary perfusion and the risk of the development of pulmonary infarction, respiratory infections and even long-term pulmonary hypertension ${ }^{5}$.

There is general agreement that patients with symptomatic severe stenosis of one or multiple PVs should be treated with PV angioplasty with or without stenting ${ }^{4,5}$. For asymptomatic patients with severe stenosis of a single PV there is no consensus on whether intervention is warranted, although the available evidence suggests that early intervention in such patients leads to clinical benefit ${ }^{5}$. In the present case, although the patient did not present symptoms, it is worth mentioning the immediate benefit that the interventionist treatment brought to the left pulmonary function, resulting in the normalization of its perfusion within 3 to 4 months after the angioplasty. Finally, it is appropriate to emphasize that the result of the PV angioplasty in the present case can only be definitively assessed after a longer follow-up period $^{8}$.

\section{Potential Conflict of Interest}

No potential conflict of interest relevant to this article was reported.

\section{Sources of Funding}

There were no external funding sources for this study.

\section{Study Association}

This study is not associated with any post-graduation program. 


\section{References}

1. Verma A, Marrouche NF, Natale A. Pulmonary vein antrum isolation: Intracardiac echocardiography-guided technique. J Cardiovasc Electrophysiol. 2004; 15: 1335-40.

2. Cappato R, Calkins H, Chen SA, Davies W, lesaka Y, Kalman J, et al. Worldwide survey on the methods, efficacy and safety of catheter ablation of human atrial fibrillation. Circulation. 2005; 111: 1100-5.

3. Noheria A, Kumar A, Wylie JV Jr, Josephson ME. Catheter ablation versus antiarrhythmic drug therapy for atrial fibrillation: a systematic review. Arch Intern Med. 2008; 168: 581-6.

4. Saad EB, Rosillo A, Saad CP, Martin DO, Bhargava M, Erciyes D, et al. Pulmonary vein stenosis after radiofrequency ablation of atrial fibrillation: functional characterization, evolution, and influence of the ablation strategy. Circulation. 2003; 108: 3102-7.

5.Di Biase L, Fahmy TS, Wazni OM, Bai R, Patel D, Lakkireddy D, et al. Pulmonary vein total occlusion following catheter ablation for atrial fibrillation: clinical implications after long-term follow-up. J Am Coll Cardiol. 2006; 48: 2493-9.

6. Dong J, Vasamreddy CR, Jayam V, Dalal D, Dickfeld T, Eldadah Z, et al. Incidence and predictors of pulmonary vein stenosis following cathete ablation of atrial fibrillation using the anatomic pulmonary vein ablation approach: results from paired magnetic resonance imaging. J Cardiovasc Electrophysiol. 2005; 16: 845-52.

7. Bunch TJ, Bruce GK, Johnson SB, Sarabanda A, Milton MA, Packer DL. Analysis of catheter-Tip (8-mm) and actual tissue temperatures achieved during radiofrequency ablation at the orifice of the pulmonary vein. Circulation. 2004; 110: 2988-95.

8. Neumann T, Kuniss M, Conradi G, Sperzel J, Berkowitsch A, Zaltsberg S, et al Pulmonary vein stenting for the treatment of acquired severe pulmonary vein stenosis after pulmonary vein isolation: clinical implications after long-term follow-up of 4 years. J Cardiovasc Electrophysiol. 2005; 20: 251-7. 